\title{
COVID-19-Related Disruptions to HIV Testing and Prevention Among Young Sexual Minority Men 17-24 Years Old: A Qualitative Study Using Synchronous Online Focus Groups, April-September 2020
}

\author{
Juan Pablo Zapata ${ }^{1} \cdot$ Madeline Dang $^{2} \cdot$ Katherine G. Quinn ${ }^{2} \cdot$ Keith J. Horvath ${ }^{3} \cdot$ Rob Stephenson $^{4} \cdot$ \\ Julia Dickson-Gomez ${ }^{5}$ Steven A. John²
}

Received: 19 March 2021 / Revised: 23 September 2021 / Accepted: 24 September 2021 / Published online: 12 November 2021

(c) The Author(s), under exclusive licence to Springer Science+Business Media, LLC, part of Springer Nature 2021

\begin{abstract}
Prior research has highlighted the impact of the COVID-19 pandemic on HIV prevention services within the U.S., but few studies have explored this impact through an exploratory, qualitative lens. In this study, we sought to highlight the voices of young sexual minority men (YSMM) 17-24 years old and explored the perceived impact of the pandemic on HIV prevention among a diverse, nationwide sample of YSMM who participated in synchronous online focus group discussions between April and September 2020. Forty-one YSMM described the negative effects of the COVID-19 pandemic on HIV testing and prevention services, including limited and disrupted access to HIV testing, HIV pre-exposure prophylaxis (PrEP), and HIV post-exposure prophylaxis. COVID-19-related challenges were compounded by ongoing, pre-COVID-19 barriers experienced by YSMM in the U.S. For instance, many YSMM relocated back home with family, causing men to avoid HIV prevention services for fear of outing themselves to relatives. YSMM also worried about placing their family at increased risk of COVID19 by attending clinical appointments. YSMM who did seek HIV prevention services, including access to PrEP, experienced significant barriers, including limited appointment availability and services not tailored to YSMM. Further efforts are needed to support YSMM re-engaging in HIV prevention during and after the COVID-19 era.
\end{abstract}

Keywords HIV $\cdot$ Pre-exposure prophylaxis $\cdot$ Sexual minority men $\cdot$ Men who have sex with men $\cdot$ COVID-19 $\cdot$ Sexual orientation

Steven A. John

sjohn@mcw.edu

1 Department of Psychology, Marquette University, Milwaukee, WI, USA

2 Center for AIDS Intervention Research, Department of Psychiatry and Behavioral Medicine, Medical College of Wisconsin, 2071 N. Summit Avenue, Milwaukee, WI 53202, USA

3 Department of Psychology, San Diego State University, San Diego, CA, USA

4 Department of Systems, Population and Leadership, School of Nursing and The Center for Sexuality and Health Disparities, University of Michigan, Ann Arbor, MI, USA

5 Institute for Health and Equity, Medical College of Wisconsin, Milwaukee, WI, USA

\section{Introduction}

On March 11, 2020, the World Health Organization (WHO) first declared the transmission of novel coronavirus SARSCoV-2, the virus which causes COVID-19, a pandemic, after the virus had spread rapidly around the world since the first reports from Wuhan, China, in December 2019 (John Hopkins Center for Systems Science and Engineering, 2021). Since then, local and state governments have tried to curb new infections and deaths by encouraging social distancing and mask wearing, which has negatively affected interpersonal relationships (Nelson et al., 2020; Sanchez et al., 2020) and access to essential services including preventive and emergency services (Richards et al., 2020). Given decades of health and social research, it is expected that changes in health care services and interpersonal functioning will disproportionally impact sexual minority men (SMM) in comparison with their heterosexual counterparts. While there is limited information on specific consequences of 
the COVID-19 pandemic on SMM, some studies show that measures of discrimination and psychological distress are pervasive and higher than observed in community samples (Kneale \& Becares, 2020). COVID-19 public health responses may also present a significant challenge for the HIV prevention and care continuums, with reduced access to HIV testing, linkage to prevention, and care services and HIV pre-exposure prophylaxis (PrEP) initiation (Ridgway et al., 2020). Because of these challenges and the COVID19 containment priority, many healthcare services have had to adapt, with telemedicine or virtual health being the most widely recognized platforms (Mann et al., 2020). With these changes, it is unclear how the COVID-19 pandemic has impacted HIV prevention and services and how it will alter future care and prevention.

SMM experience a disproportionate burden of HIV incidence in the USA, accounting for $69 \%$ of new infections in 2018 with significant disparities by race and ethnicity (CDC, 2018). Black or African American and Hispanic or Latinx SMM account for 37\% and 30\% of new infections, respectively (CDC, 2018). Additionally, there are age group differences, with individuals 13-24 years old accounting for 25\% of new infections among SMM (CDC, 2017a). In order to remain knowledgeable about their HIV status and congruent with Centers for Disease Control and Prevention (CDC) recommendations (CDC, 2017b), individuals often rely on regular testing at local clinics and medical offices, many of which decreased their patient load or closed at the start of the pandemic. Since these services became limited beginning in March 2020, there was a significant reduction in testing among SMM (Stephenson et al., 2021).

Simulation modeling indicates the potential for increased HIV incidence attributable to disruptions in HIV prevention services. Jenness et al. (2020) simulated the projected incidence of HIV among SMM in two scenarios over an 18-month period after the start of the pandemic. In their study, scenario one was simulated based on if individuals exhibited a reduction in sexual behavior concurrent with a reduction in sexual health services. The second simulation was a scenario in which SMM did not change their sexual behavior but did experience a reduction in sexual health service utilization. In scenario one, they found that there would be an overall reduction in HIV incidence, where decreased sexual encounters offset the decreases in clinic availability. In scenario two, there was an overall increase in HIV incidence attributable to decreased clinical services. Between March and May 2020, researchers reported SMM 14-17 years old engaged in fewer in-person sexual encounters and identified a $10 \%$ increase in virtual/video sex (Nelson et al., 2020). Researchers also identified a reduction in sexual behavior among SMM between February and June 2020, but there was also a decrease in availability for HIV services including access to PrEP (Pampati et al., 2021), aligning with reports of diminished access to HIV post-exposure prophylaxis (PEP) (Junejo et al., 2020).

In 2021, almost a decade prior to the start of COVID-19, the US Food and Drug Administration approved the use of PrEP for HIV prevention (U.S. Food and Drug Administration, 2012), adding another biomedical tool to combat the HIV epidemic beyond treatment as prevention and PEP. Whereas non-occupational PEP is a three- or four-drug regime of antiretrovirals administered within $72 \mathrm{~h}$ after a potential HIV exposure recommended by the CDC since 2005 (CDC, 2017c; Smith et al., 2005) to reduce the risk of HIV seroconversion (Mayer et al., 2017), PrEP in its current form is a once-daily pill regime supported by CDC prescribing guidelines to be taken before HIV exposure events (CDC, 2017c). While fewer than 12\% of HIV-negative or unknown status SMM have reported taking PEP in their lifetime (John et al., 2021), there was a $470 \%$ increase in PrEP uptake in individuals over 16 years old, predominantly among cisgender men, between 2014 and 2016 (Ya-lin et al., 2018). Nonetheless, fewer than one-in-four SMM (inclusive to both cisgender and transgender men) reported taking PrEP, with significantly fewer Black and Latinx SMM reporting current PrEP use compared to white SMM (Holloway et al., 2017; John et al., 2019; Zarwell et al., 2020). CDC guidelines recommend HIV/STI testing at quarterly intervals for clinicians to refill PrEP (CDC, $2017 \mathrm{c}$ ), and this has been identified as a major area of burden among SMM during COVID-19 (Sanchez et al., 2020).

Early surveys of SMM indicated major disruptions to sexual health services resulting from the COVID-19 pandemic. In one study, $22.6 \%$ of participants $15-24$ years old reported difficulty obtaining HIV testing (Sanchez et al., 2020). Additionally, another study found that among SMM currently prescribed PrEP, 32\% of their study population did not have an HIV test in the previous three months, with $20 \%$ reporting difficulty obtaining a test (Pampati et al., 2021). To curb these challenges to obtaining HIV testing and prevention services during COVID-19, the CDC recommended clinicians order home-collected samples from approved laboratories, prescribe 90-day PrEP supplies instead of the regular 30-days, and offer telehealth services if possible or refer to other clinics that have the capabilities (CDC, 2020). The CDC also recommended oral swab HIV self-testing as an alternative option with the caveat of its lower sensitivity for detecting acute infections (CDC, 2020). Nonetheless, only brief accounts of the impact of COVID-19 have been reported to date, with few studies exploring the impact of the pandemic through an exploratory, qualitative lens. As such, we sought to highlight the voices of young SMM (YSMM) 17-24 years old and explored the impact of the pandemic on HIV prevention among a diverse, nationwide sample of YSMM who 
participated in online focus group between April and September 2020.

\section{Methods}

\section{Participants and Procedures}

As described previously (Hong et al., 2021; screening data), participants were recruited online from social media and men-for-men geosocial networking apps between March and September 2020 to participate in one of nine online synchronous focus group discussions (FGDs) about their general experience with HIV prevention during COVID-19. Primary recruitment images featured two men, with young couples of various race/ethnicities across images designed to oversample Black and Latinx YSMM. Fraudulent responses to the screening survey were minimized by excluding any information on eligibility criteria from study advertisements and referral mechanisms, using the "prevent ballot box stuffing" feature in Qualtrics to prevent multiple responses, offering no incentive for completion of the brief ( $\sim 5$ to $10 \mathrm{~min}$ ) screening survey and its associated online consent procedure, and using a delayed invitation procedure for the online FGDs to avoid attempts at determining the study's eligibility criteria (Teitcher et al., 2015). To further ensure data integrity, duplicates were checked using a procedure of comparing contact information (i.e., name, email, phone number) and IP addresses.

To be eligible to participate in an online FGD, participants were required to: (1) be 17-24 years old; (2) identify as male (including transgender men); (3) report one or more male sexual partners in the past 6 months, including those who identified as transgender; (4) self-report HIV-negative or unknown status; (5) report sexual behavior meeting CDC guideline criteria for PrEP (CDC, 2017c), which included the past- 6 month behavior of recent bacterial sexually transmitted infection, condomless anal sex (CAS) with a casual male partner, CAS with an HIV-positive or unknown status main partner, or CAS with an HIV-negative main partner who reports CAS with other male partners; and (6) reside in the US. Individuals who screened eligible received an email invitation to participate. Eligible participants who replied to our email invitation were then asked to complete an online consent procedure. Agreement-to-participate was obtained through a guided procedure using Qualtrics that described the study's purpose, procedures, and other critical components. Participants were encouraged to email or call to get clarification on any questions prior to continuing, and several participants emailed with questions about privacy protections. Participants then completed a brief quiz as a capacity-to-consent procedure to ensure adequate comprehension of the critical components of consent, including the voluntary nature of the study, risks and benefits to participation, and confidentiality of all data collected. Participants then agreed to participate online, and a copy of the study's informational letter was emailed to the address of their choosing. A waiver of guardian permission was obtained for those considered minors. Participants were then scheduled for upcoming online group chat(s), with 6-12 individuals invited per group.

Synchronous online focus groups were administered by a team of 2-3 researchers via online chat. Specifically, we used the real-time web-based meeting client Adobe Connect for the online group chats, which allowed between-participant anonymity with pre-selected usernames during consent and no video recording. This method for conducting focus groups has been found to increase discussion regarding sensitive topics while maintaining fidelity of themes identified compared to in-person focus group discussions previously (Reisner et al., 2018; Woodyatt et al., 2016). Online focus groups were about $90 \mathrm{~min}$ in duration, and participants were compensated with a $\$ 40$ e-gift card. All text data were saved for analysis. All study procedures were approved by the Institutional Review Board of the Medical College of Wisconsin.

\section{Focus Group Content}

Using a semi-structured focus group guide, we sought to understand barriers and facilitators to increase access to HIV testing in the context of the COVID-19 pandemic. Content areas included (1) general healthcare utilization patterns and behaviors prior to COVID-19, (2) impact of COVID-19 on HIV testing throughout stages of the pandemic, (3) attitudes about and experiences with biological approaches to HIV prevention (i.e., PrEP, PEP), (4) perceived barriers to HIV testing and biomedical HIV prevention, and (5) adapted virtual health care initiatives for sexual minority men. We explicitly asked about direct occurrences of healthcare engagement before and during the pandemic and new health care initiatives to inform effective and adaptive resources in a post-pandemic environment. Example semi-structured interview guide questions applicable to this analysis included: "If you wanted to get PrEP right now, how do you think COVID19 would impact your ability to get a prescription? What would you do?" and "If you wanted to get PEP today, tell us how you would do that given the impact of COVID-19?".

\section{Data Analysis}

Descriptive statistics were used to characterize the sample using screening survey data. Transcripts were initially coded using MAXQDA, a qualitative analysis software using a combination of deductive and inductive coding to categorize the data (Saldana, 2014). Codes were identified by the first author, trained in qualitative methods using a three-stage analytic coding strategy including open, axial, and selective coding (Corbin \& Strauss, 1997). First, a list of a priori codes 
was developed in advance by the first and last author on topics addressed by the focus group guide. Codes were created by noting overlapping themes in the transcripts and developing code definitions that represented the data. Each transcript was coded and reviewed separately to ensure adequate application of codes. During the initial analytic phase, each analyst separately coded the same randomized transcript with the final codebook and inconsistencies were discussed until agreement was reached. The final codebook included barriers and facilitators to HIV testing and prevention efforts during the COVID-19 pandemic and changes in sociodemographic characteristics (including housing, relationship status, rural/ urban setting and differences in health care). Coded focus groups were then analyzed using thematic content analysis (Braun \& Clarke, 2006) to highlight patterns and identify meaning of the data by the first and senior author.

\section{Results}

Recruitment activities identified 133 YSMM eligible for our online focus groups. Of the 118 who provided contact information and were invited to participate, 55 consented and 41 participated in an online FGD. Nine FGDs were conducted spanning April-September 2020, with 3-7 participants in each group. Focus group participants were predominantly $(85.4 \%)$ cisgender men and self-identified as gay $(65.9 \%)$ or bisexual (29.3\%). Average age of participants was 21.0 years, with $36.6 \%$ of the sample being under 21 years of age and five participants $(12.2 \%)$ who were 17 years old. Sample majority was Black (26.8\%) or Latinx (29.3\%), and about half (53.7\%) reported their relationship status as single. Participants were geographically represented from all four US Census regions, and $78.1 \%$ were recruited from social media. By PrEP use status, $22.0 \%$ of participants were currently taking PrEP, and $17.1 \%$ were former PrEP users. Three participants $(7.3 \%)$ reported prior PEP use in their lifetime. Full sample characteristics are provided in Table 1.

Excerpts from the nine focus groups are used below to demonstrate four primary themes: (1) HIV prevention engagement before COVID-19, (2) impact of COVID-19 on HIV testing, (3) biomedical HIV prevention, and (4) changes in housing status and income during the pandemic.

\section{HIV Prevention Engagement Before COVID-19}

In an effort to characterize how the COVID-19 pandemic has impacted HIV prevention, we initially sought to understand how YSMM experienced accessing preventive health care prior to COVID-19. Although extensive research has documented HIV health care services among YSMM (Quinn et al., 2019; Siegler et al., 2018; Zapata et al., 2020), it was important for us to document preventive health care engagement as a form of comparison to the impact of the pandemic on HIV prevention. Community and personal health were often central to HIV prevention, as young men described the need to test frequently for HIV and seek LGBT affirmative health care services with supportive providers.

FG 1; P1: Getting tested for HIV is extremely important, not only for my own personal health but to protect those that I am with sexually. Also, HIV symptoms can be dormant for months or years, so it's important to be tested once every three months to be safe and stop the spread.

FG 5; P2: HIV testing can prevent unmitigated spread of the virus in our communities. If I test positive, I can inform previous partners and improve my safer sex practices with future partners.

These sentiments were raised and discussed in other groups and were consistent throughout. Participants felt they were informed about the potential risk of HIV infection, and how they could be actively involved in testing and education in order to reduce their personal risk. Yet, they described ongoing barriers to access, in both HIV testing and PrEP. While young men generally were able to access these services, they also described significant challenges or perceived barriers, in which they were not immediately able to get tested or get on PrEP, were treated unfairly, and/or were unwilling to seek services. This was evidenced by experiences shared by other participants.

Interviewer: What are some reasons why getting tested for HIV is important? What barriers to HIV testing have you or others experienced?

FG 3; P4: Some barriers and deterrents for testing would probably be the stigma around being positive, homophobia in healthcare, and the shame factor - especially if you're having unprotected sex or have a lot of sex partners. Also, people in rural and/or more conservative areas may struggle to get access to low-cost testing.

FG 1; P3: I have experienced discrimination from doctors when I have been tested for HIV. I was told I "needed to stop doing this to myself" (referring to having unprotected oral sex with men) after going to the same Urgent Care to get tested after a couple of times.

Descriptions of mistreatment and inadequate access frequently included experiences of discrimination, lack of transportation, medical mistrust, anxiety, the availability of quality and affordable community-based care, and homonegativity. For example, much like described above, another participant described fear about disclosing his sexual orientation to his provider because he did not want his doctor or family to find out. Similarly, another participant discussed reduced access to health care because he was still on his 
Table 1 Demographics characteristics of young sexual minority men $(n=41)$

\begin{tabular}{|c|c|c|}
\hline Continuous variables & $M$ & SD \\
\hline Age (range 17-24) & 21.0 & 2.5 \\
\hline Categorical variables & $N$ & $\%$ \\
\hline \multicolumn{3}{|l|}{ Gender identity } \\
\hline Cisgender man & 35 & 85.4 \\
\hline Transgender man & 6 & 14.6 \\
\hline \multicolumn{3}{|l|}{ Race/ethnicity } \\
\hline Black, non-Hispanic & 11 & 26.8 \\
\hline Latinx or Hispanic & 12 & 29.3 \\
\hline White, non-Hispanic & 14 & 34.2 \\
\hline Multiracial/another & 4 & 9.8 \\
\hline \multicolumn{3}{|l|}{ Sexual orientation } \\
\hline Gay & 27 & 65.9 \\
\hline Bisexual & 12 & 29.3 \\
\hline Queer & 2 & 4.9 \\
\hline \multicolumn{3}{|l|}{ Relationship status } \\
\hline Single & 22 & 53.7 \\
\hline Partnered & 19 & 46.3 \\
\hline \multicolumn{3}{|l|}{ Pre-exposure prophylaxis (PrEP) use status } \\
\hline Never & 25 & 61.0 \\
\hline Prior PrEP use & 7 & 17.1 \\
\hline Current PrEP use & 9 & 22.0 \\
\hline \multicolumn{3}{|l|}{ Post-exposure prophylaxis (PEP) use history } \\
\hline Never & 38 & 92.7 \\
\hline Prior PEP use & 3 & 7.3 \\
\hline \multicolumn{3}{|l|}{ Region } \\
\hline Midwest & 12 & 29.3 \\
\hline Northeast & 11 & 26.8 \\
\hline South & 12 & 29.3 \\
\hline West & 6 & 14.6 \\
\hline \multicolumn{3}{|l|}{ Recruitment source } \\
\hline Social media & 32 & 78.1 \\
\hline Men-for-men geosocial networking apps & 9 & 22.0 \\
\hline
\end{tabular}

Percentages may not add up to 100 due to rounding

parent's health insurance and they did not know about his sexual orientation. Some young men discussed how, because of their racial identity and the deep rooted homonegativity in the Black community, they were unequipped to navigate their sexual health or were fearful of being further discriminated against by their own community.

FG 9; P6: Well, the black community has a distorted ideal on what masculinity and manhood is. It's a straight man only. However, gay is seen as feminine. There's no way I can be a black man and a gay man at the same time.

In addition to social and cultural challenges, there were a significant number of young men who discussed negative perceptions and stigma related to PrEP. Such attitudes have shaped norms about HIV health care, the acceptability of these services, and as a result, a lack of engagement with PrEP. Participants in focus groups three and five discussed discrimination from their provider, which has continued to impact their patient-provider interactions.

FG 3; P7: I went to an Urgent Care and the nurse and doctor both ridiculed me on my "lifestyle" and number of partners and the fact that I came in every 3 months to get tested.

FG 5; P8: I would be afraid of being discriminated against by my healthcare provider because of my orientation. Some healthcare providers have said they will 
not cover bisexual men for PrEP or HIV related medication because they also have sex with women.

In every group, at least a few participants had similar experiences of being mistreated that limited their ability to access HIV-preventive services. It is important to highlight that regardless of these experiences, many of the men we interviewed felt a responsibility to access prevention services to protect their own health and the health of their communities and were able to navigate these barriers and find HIV prevention out of their own accord.

\section{Impact of the COVID-19 Pandemic on HIV Testing}

Throughout each focus group, participants described restrictions that created significant barriers to accessing HIV testing services. Such experiences shaped the availability of quality and LGBT affirmative community-based care, with more severe and persistent challenges for Black and Latinx men. In light of federal, state, and local orders to socially isolate in order to reduce the risk of COVID-19, a significant number of men in this study reduced their vulnerability to HIV as a result of decreased occurrences of sexual behavior.

FG 1; P1: I rather stay home and not have sex than risk getting COVID-19.

FG 1; P2: Depends who you are, but I am no longer sexually active because I am living at home with parents and staying home.

FG 1; P3: I won't risk getting a virus from going to get tested for another one.

There was a similar discussion in focus group two:

FG 2; P4: I wouldn't get tested; I would wait until COVID settles down.

Interviewer: How do people weigh the risk of HIV with COVID-19?

FG 2; P5: Now definitely wouldn't be the time to be going out a lot or increasing your sexual network. Both in terms of transmitting/catching HIV and transmitting/ catching COVID.

Public health ordinances mandated closure of non-essential businesses to minimize the risk of COVID-19 within communities. Participants in all groups reported that their sexual network became smaller-primarily within the earlier focus group interviews conducted in April and June. It is important to highlight that this was for several reasons. First, many participants discussed the very real and possible threat of COVID-19 and did not want to risk "one virus" to test for another "virus" or potentially being exposed from sexual partners. For those who were willing to engage in continued sexual activity during the pandemic and/or test for HIV, some discussed the secondary fear and guilt they felt as a result of their potential exposure:

Interviewer: Has COVID-19 changed your sex lives much? If so, how?

FG 4; P5: I also feel guilty during the times that I do hookup w/ other guys via apps like Grindr and Jack'd just b/c I have the fear of catching COVID and spreading it.

FG 6; P6: I haven't tried to get tested since the pandemic started and I wouldn't feel comfortable entering the clinics/offices during this time anyways.

FG 5; P7: Since COVID, leaving the house to meet guys has been such an anxious and scary thing to do.

FG1; P8: As for changes with COVID-19, I am not as sexually active as I was before this all started.

Furthermore, some men also discussed meaningful changes to current HIV testing and counseling services and provider interaction(s). For instance, one participant in focus group seven expressed that he was told not to come into the hospital for HIV-related services:

FG 7; P9: I tried to get tested a couple months ago at the clinic and the nurse told me that it would be better not to go because the pandemic.

Other participants in other focus groups described the impact of COVID-19-related outpatient clinic restrictions on their intent to receive voluntary HIV testing:

Interviewer: How has COVID-19 impacted your ability to get HIV testing?

FG 4; P10: omg getting it was such a production. I went to this place called Red Door clinic in the Twin Cities, but I wasn't "high risk" enough, so they basically said sorry you wasted your time try Planned Parenthood instead.

FG 1; P11: Doctors are trying to reduce the number of patients coming in person unless absolutely necessary. I haven't tried to go get tested, but knowing this, I feel they would turn me away if I just wanted to get a test

FG 1; P12: I had to do more to get tested, testing centers are more appointment only, and there's less transportation available to appointments, and so on.

FG 2; P13: It has impacted my ability to test because doctors' offices are either flooded [with patients] or closed.

FG 6; P14: I've tried to get tested but the center I usually go to was not accepting appointments

Although several participants in the groups understood why clinics might need to take additional precautions to reduce the spread of COVID-19, they still expressed concern about their individual risk for HIV. Context was also important; for YSMM in rural settings these challenges 
were compounded by significant changes to their housing and transportation.

FG 5; P15: I live in a conservative rural community and have to drive a long way to a supportive care facility, I just choose to not take PrEP at all right now because of the frequent required visits.

FG 7; P15: Back home I live in a rural and conservative community, I would need to go to the city to get tested and it would need to be a safe place for me.

It is important to note that some of the larger, more urban-based community clinics were able to accommodate for COVID-19-related challenges and were thus able to resume HIV testing and counseling. For example, for those clinics that were able to continue their services, they were able to implement the use of HIV self-testing and telehealth as pivotal mechanisms to provide evidence-based services.

FG 5; P16: My clinic has reduced testing and doesn't do in person visits. However, they've continued testing on a request basis, and as always, we do our own swabs and samples, just submit them.

FG 7; 17: I went to a different location because my doctor's office was closed due to COVID. I wore a mask the whole time, it was very socially distanced. They gave me a self-testing kit and told me on the spot.

FG 8; P18: My clinic is still doing remote (Zoom) appointments at the moment (as far as I'm aware), but then they have us come in to have samples collected for testing with special precautions.

As with any new health care initiative, some noted challenges with virtual appointments. For example, one participant in focus group four discussed logistical issues with his virtual appointment for HIV testing:

FG 4; P19: The logistics were really frustrating with the virtual visit because I think they were still working out the kinks. They cancelled on me 30 minutes after my appointment was supposed to start, and then didn't have availability for about a week after that.

As such, lack of in-person care presented challenges to continuing HIV prevention, further compounding prepandemic challenges with HIV prevention accessibility. Unfortunately, the need for these testing services is unchanged. Interestingly, there were gradual changes in risk, such that in the initial focus groups conducted during the beginning of the quarantine in April, there were notable decreases in sexual relationships reported by participants, but men in FGs in August and September 2020 reported similar sexual behavior before the pandemic began.

\section{Impact of the COVID-19 Pandemic on Biomedical HIV Prevention}

In addition to significant challenges with HIV testing and counseling, PrEP services were also disrupted both in maintenance and initiation. Although several participants were on PrEP, they experienced barriers to attending appointments, receiving refills, and/or obtaining their quarterly testing. Men in focus groups three and five, for example, discussed how they had to discontinue their PrEPuse because of the pandemic.

FG 3; P1: I couldn't get PrEP filled because my appointment was cancelled because of the pandemic. FG 5; P2: When I feel comfortable to start having casual sex again, I will restart PrEP and get the standard battery of STI tests again, including restarting regular HIV testing.

In addition to men who were already on PrEP, some men who were possibly interested in initiating PrEP services discussed social and structural barriers to PrEP and healthcare. Many focus group participants who had to relocate back to their family homes because of the pandemic were reluctant to start PrEP, often citing their parents' disapproval of their "lifestyle" or not being "out" to their immediate family.

FG 6; P3: I think starting PrEP would be difficult now, depending on the amount of initial testing one would need. I also think that it could be difficult to do a telehealth appointment in a household that one is not out to.

FG 1; P4: I have never taken PrEP. What concerns me most about PrEP right now is that I had to move back home, and I can't hide it.

These experiences were compounded by the immediate and ongoing threat of COVID-19. It is thus possible to suggest that due to the impact of COVID-19 on an alreadystrained health system, PrEP services have been further pushed back as a result of both fear and structural challenges.

Interviewer: Tell us more about why you stopped taking PrEP during COVID-19.

FG 5; P6: I'm not having as much casual sex because I live with older family now. For me it wasn't an issue to stop this to mitigate my bringing more COVID risk in our home. If I lived alone or with other young healthy friends, I might still be having sex and taking it.

These challenges also extend to a similar biomedical prevention tool, PEP. As was previously noted, PEP requires immediate intervention to be most effective. Unfortunately, given the added restrictions due to the pandemic, it may be more difficult to access this method in an emergency setting. 
FG 1; P7: Correct me if I am wrong, but I understood that PEP could also be obtained in emergency rooms. Currently that would be very difficult given the COVID-19 pandemic.

FG 6; P8: Could I go the ER for PEP during COVID? or would I just get judged since people are even more ashamed to ask for PEP than they are for PrEP. There is often more shame in having a risky sexual encounter. FG 8; P9: I probably could not get PEP on time for it to work during COVID.

FG 8; P10: I really would not know where to start if I wanted to get PEP during COVID. I would be too embarrassed to go into a hospital for that during a pandemic.

FG 9; P11: I would be concerned about the PEP window-period and getting it on time with all of the hospital changes because of COVID.

Moreover, according to a participant in focus group six, he had initial interest in beginning to take PrEP, but because of both reduced access to a community-based clinic and sexual stigma, he ultimately decided to "postpone" his decision to begin PrEP until COVID-19 restrictions are lifted. This conversation reflects the underlying conflict in navigating cooccurring epidemics and how such interactions can impede the successful dissemination of preventive services.

\section{Changes in Housing Status and Income during the Pandemic}

It is important to highlight the severe socioeconomic impact experienced by some gay men and other SMM due to COVID-19 and the response to this pandemic. Responses in our focus group indicated that there were substantial changes in income and housing that directly and indirectly affected HIV testing and access to other preventive services, such as PrEP and PEP. Our data indicate that some respondents had to relocate back home with their immediate or extended family to combat the financial strain of the COVID-19 pandemic.

FG 3; P1: Since I moved back home, the main barriers have been getting to a testing center without my parents knowing and also general transportation to the testing center.

FG 6; P2: I can't easily lie to my parents about where I'm going and then get tested. I almost never leave the house, so I can't get tested at all. It was so easy to get tested before COVID-19 when I didn't live at home all the time.

These young men highlighted an important issue. Participants reported (or felt that) they were at heightened HIV risk as SMM, yet they are not readily able to access preventive resources because of changes in their housing, as noted throughout some FGDs. In every group, at least a few participants had similar experiences of having to move back home since the beginning of the pandemic, and there was nearly universal recognition that such experiences affected their ability to access HIV testing for a number of reasons. For instance, in focus group eight, one participant noted that their family was unaware that he was sexually active, and therefore would not be able to get tested without having to hide it to some extent since he now lived at home with family. Participants also generally noted they were unable to access testing or engage in sex because they did not want to place their family members at heightened risk for COVID-19 by going to the clinic or expanding their protection bubble.

FG 7; P3: If I was in dire need of a test, I would have to tell my parents that I was going out to get tested. I have a 2- and 7-year-old in the house so we are being very cautious with COVID19. I suppose I could at-home test, but I have never done one before and wouldn't know where to get one.

FG 4; P4: I now live w/ my mom who is 63 (and it has been said that older people can't fight corona off as easily as younger people).

FG 8; P6: Yeah, I have messaged with guys a little when I am bored but if they start actually trying to invite me over or something, I usually stop messaging because my family is taking COVID pretty seriously.

As demonstrated in these responses, there are significant barriers to accessing HIV testing and counseling for young men who relocated due to the pandemic. In addition to changes in housing, there were negative economic impacts experienced by men due to COVID-19, which had substantial effects on PrEP use decision-making. For instance, many men in our sample reported loss of employment and anticipated reductions in income, and cost is a well-established structural barrier to PrEP and the auxiliary clinic services required (e.g., quarterly HIV/STI testing). This is further demonstrated in focus groups five and nine:

FG 9; P7: Without insurance PrEP would be $2 \mathrm{k}$ a month which is insane, and I can't do that right now.

FG 5; P8: I took PrEP until the start of the pandemic. Truvada. The cost is ridiculous without insurance. I still have my dad's killer insurance, otherwise I'm not sure the manufacturer coupon would be enough to afford it especially without a job anymore.

YSMM highlighted the consequence of moving away from colleges and universities on HIV-preventive services as a result of the pandemic. Some men in our study were dependent on affirmative and more affordable health care services offered by their school, and given the closures of these spaces, many men were then unable to readily access testing or PrEP-related services they were accustomed to receiving. 
FG 7; P9: My college not offering testing made it more expensive and cumbersome, but thankfully I have insurance. I realized however, how much I depended on my college's clinic. They can even prescribe me with PrEP. FG 9; P: 10: Haven't gotten HIV tested since March when school shut down and I moved back home due to COVID.

Further compounding the cost barriers associated with PrEP are the direct and indirect consequences of the COVID19 pandemic. Some individuals in this study did not have access to their own medical insurance, had to relocate, or had their incomes reduced, which were significant barriers to continuing or initiating PrEP. As such, only eight of the 41 participants (22\%) were currently using PrEP, despite everyone in the sample meeting CDC criteria for PrEP.

\section{Discussion}

The purpose of our study was to determine the perceived impact of the COVID-19 pandemic on HIV prevention among a U.S. nationwide sample of HIV-negative or unknown status YSMM, with an additional aim to understand barriers and facilitators to HIV testing and prevention during the pandemic. Notable advances have been achieved with the introduction of PrEP and other prevention efforts toward reducing HIV incidence, but the COVID-19 pandemic threatened to undermine progress by exacerbating existing barriers to HIV testing. As COVID-19 continued to spread around the world for more than a year at the time of this paper, it is likely that the pandemic and the response to it will influence these disparities and access to HIV-preventive services. It was evident in our focus groups that the COVID-19 pandemic, to date, caused significant challenges for HIV prevention that extended beyond changes due to quarantine and community containment measures. Rather, much of these young men's challenges to HIV testing and prevention was rooted in existing structural barriers (e.g., lack of transportation, poverty, location of biomedical resources) and experiences of, or anticipated, rejection from family. This study was therefore intended to provide a guide for future intervention amidst global changes and challenges from COVID-19 and to serve as a foundation for additional research.

As has been documented in previous research, medical mistreatment, inadequate access, HIV-related anxiety, and homonegativity contributed to structural and cultural barriers that make it difficult for YSMM to access HIV-preventive services (Doll et al., 2018). As demonstrated in each focus group, these barriers were exacerbated by the COVID-19 pandemic. For instance, throughout each focus group, there were general changes to community-based care and HIV-preventive clinics, such as closures and changes to services (e.g., virtual appointments, reduced capacity, and at-home testing). These findings are consistent with published research reporting disruptions to HIV and sexual health services in the USA during the pandemic (Junejo et al., 2020; Pampati et al., 2021; Ridgeway et al., 2020; Stephenson et al., 2021).

YSMM discussed COVID-related fear as an additional barrier for HIV testing. As one participant noted, he would, "not risk a virus from going to get tested for another one." As expected, some men also discussed meaningful changes to current HIV testing and counseling services and provider interactions. Compared to previous HIV testing experiences, in-person testing in clinics and emergency rooms were significantly limited or impossible as "routine" testing was not recommended given the heightened risk of COVID-19. Consequently, some men described that they were either turned away from the clinic or had to seek additional services in other settings as a result of ongoing changes to the health care system. It has been well documented that most new HIV transmissions are linked to people who become newly infected but are unaware of their HIV-positive status (Skarbinski et al., 2015). These challenges were magnified as the COVID-19 pandemic impacted these pivotal prevention mechanisms.

Challenges resulting from the COVID-19 pandemic also extended to biomedical prevention including access to PrEP and PEP. Although extensive measures were put in place to accommodate COVID-19-related changes in PrEP-use, men noted various challenges in connecting with PrEP and PEP during the pandemic. It was well documented since before the pandemic that telehealth could be a suitable mechanism to connect people with PrEP (John et al., 2017; Touger et al., 2019), yet as discussed by men in our study, there were notable barriers to virtual appointments. For instance, some men described that it was impossible to attend a virtual appointment for HIV prevention, as they did not have the necessary privacy to safely discuss sexual behaviors. As PrEP requires ongoing testing for prescription, it was also noted that due to changes in their addresses and moving in with relatives who may not approve or know of their sexuality, they were not able to attend their typical appointments and instead, for example, were going to wait until they were able to move back to their college-setting where culturally competent care was readily accessible. Our results align with patient and clinic-based reports of reductions in HIV and STI testing during the pandemic (Hill et al., 2021; Stephenson et al., 2021), and while telehealth was successfully used to support continued PrEP use for some during the pandemic (Hill et al., 2021; Rogers et al., 2021), our data lead us to believe YSMM may have suffered disproportionately from disruptions to clinicbased care given their unique living circumstances and confidentiality concerns.

Our data highlight the significant changes in housing experienced by SMM in our study due to COVID-19 and 
the response to it. Many men had to relocate back home with their families during the pandemic and reported losses in income. These changes indirectly affected access to HIV testing and PrEP-use by underscoring existing structural and cultural barriers to health care. Many have also raised the alarm that they were unaware of alternative HIV-preventive services, such as where to access PEP, which are novel strategies to decrease the prevalence of HIV. Indeed, we show that this pandemic impeded uptake of HIV testing in areas already most impacted by lower access to affirmative health care services. These circumstances were further compounded by YSMM relocating back home to live with families, which created new barriers to alternatives of virtual-based appointments because of concerns about disapproval or outing themselves to their family. These findings align with reports from younger, adolescent SMM aged 14-17, who experienced added burden and deleterious impact on mental health from stay-at-home orders because of issues associated with privacy and confidentiality related to their sexuality (Nelson et al., 2020).

Young men in this study also expressed an overwhelming burden of additional work to receive HIV-related servicesunique findings not previously reported. For instance, one participant noted that he had to schedule several appointments and had to visit three clinics in order to be seen for PrEP. Another participant described how his appointment was canceled due to COVID-19 and had to wait several weeks for an additional appointment. For men without access to transportation and who fear rejection, HIV-preventive care was near impossible given the growing threat of COVID19 infection and the global response to it. Thus, it is not surprising that young men would decide to postpone their decision to test for HIV or initiate PrEP. The data presented here demonstrate how COVID-19 may lead to a spike of HIV infection, especially coupled with data reporting reengagement of sexual activity as the pandemic extended onward and barriers to HIV prevention services in other samples of SMM (Pampati et al., 2021; Stephenson et al., 2021). Moreover, prior reports documented disruptions to biomedical HIV prevention during the pandemic (Hong et al., 2021; Junejo et al., 2020; Pampati et al., 2021; Stephenson et al., 2021), and our findings uniquely illustrate with their voices the challenges some patients faced seeking affirming HIV prevention services during the middle part of 2020 amidst the COVID19 pandemic.

There have been recent efforts in the USA to attempt to incorporate advances in HIV-preventive and PrEP services to respond to COVID-19 (Shoptaw et al., 2020) in order to limit the gap of HIV testing during the pandemic. Some of these strategies were identified by men in our FGDs, such as the use of HIV self-testing and mobile outreach services during various stages of the pandemic. Yet, these initiatives require significant investment and infrastructure and may be difficult to implement within communities that have reduced access to care and are less informed and/or supported. As previously discussed, there was a universal decrease in HIV healthcare utilization, and while COVID-19 vaccination efforts have improved these outcomes throughout the USA, it is likely that there will be longstanding challenges to HIV care and prevention (Pampati et al., 2021; Stephenson et al., 2021). As noted in our FGDs, many YSMM received care through telehealth, and recommendations for future research are to develop a metric that will measure and guide virtual care improvement, delineate for whom telehealth helps improve access and any indirect consequences of expansion post-pandemic, and removal of insurance reimbursement barriers built on a framework of clinic-based care. Additional research is also recommended to improve health disparities among people of color and rural communities lacking proximity to a health center in their community. This may include special provisions for patients who lack access to the internet or confidential space. There may also be opportunities to enlist local communities in supporting HIV self-testing, given that many YSMM noted barriers at home that limited their ability to engage in home-based HIV testing. Nonetheless, the COVID19 pandemic has allowed for rapid expansion of innovation to support HIV prevention and care delivery (Armstrong et al., 2021), yet further efforts are needed to support these initiatives for long-term benefit.

\section{Limitations}

Our research is not without limitation. First, we only used online strategies for recruitment and data collection as an ethical way to minimize the risk of COVID-19 among our participants; as such, generalizability of our findings should be limited to YSMM with internet access. Nonetheless, both recruitment and online focus group procedures were accessible for individuals with smartphone-only internet access, whereby reducing some access concerns. No participants had connectivity issues that could not be resolved. Second, focus groups were conducted using a chat-based format, which could have limited focus group facilitators' ability to use non-verbal cues to guide additional probing. However, we believe this effect to be minimal regarding the identification of themes when compared to in-person FGDs (Reisner et al., 2018; Woodyatt et al., 2016).

\section{Conclusion}

Forty-one YSMM described the negative effects of the COVID-19 pandemic on HIV testing and prevention services, including limited and disrupted access to HIV testing, PrEP, and PEP. COVID-19-related challenges were compounded by ongoing, pre-COVID barriers experienced by YSMM in the USA. For instance, many YSMM relocated back home with 
family, causing men to avoid HIV prevention services for fear of outing themselves to their families. YSMM also worried about placing their families at increased risk of COVID-19 by attending clinical appointments. YSMM who did seek HIV prevention services including access to PrEP experienced significant barriers, including limited appointment availability and incompetent services for YSMM. As such, further efforts are needed to support YSMM re-engaging in HIV prevention during and after the COVID-19 era.

Acknowledgements The authors acknowledge the contributions of other members of the study's advisory team, especially Drs. Jeffrey Kelly, Andrew Petroll, Jennifer Walsh, H. Jonathon Rendina, and Christian Grov. We wish to thank our staff at the Center for AIDS Intervention Research at Medical College of Wisconsin, especially Karen Opgenorth, Kevin Brown, Tom Lytle, Thom Ertl, and Erika Christenson. We also wish to thank our participants who volunteered their time.

Funding Funding support was provided by the National Institute of Mental Health (K01-MH118939, PI: John; K01-MH112412, PI: Quinn) and the National Center for Advancing Translational Sciences (UL1TR001436; TL1-TR001437). The content of this manuscript is solely the responsibility of the authors and does not necessarily represent the official views of the National Institutes of Health.

Data Availability Data are not accessible to the public to minimize the risk of loss of confidentiality.

\section{Declarations}

Conflict of interest All authors declare that they have no conflict of interest.

Ethical Approval All procedures performed in studies involving human participants were in accordance with the ethical standards of the institutional and/or national research committee and with the 1964 Helsinki Declaration and its later amendments or comparable ethical standards.

Informed Consent This study met the Medical College of Wisconsin Institutional Review Board's definition of "minimal risk," and a waiver of informed consent was granted. All participants agreed to participate after completion of a guided procedure using Qualtrics that described the study's purpose, procedures, and other critical components, as well as a capacity-to-consent procedure. A waiver of guardian permission was obtained for those considered minors.

\section{References}

Armstrong, W. S., Agwu, A. L., Barrette, E. P., Ignacio, R. B., Chang, J. J., Colasanti, J. A., Floris-Moore, M., Haddad, M., MacLaren, L., \& Weddle, A. (2021). Innovations in Human Immunodeficiency Virus (HIV) Care Delivery During the Coronavirus Disease 2019 (COVID-19) Pandemic: Policies to Strengthen the Ending the Epidemic Initiative-A Policy Paper of the Infectious Diseases Society of America and the HIV Medicine Association. Clinical Infectious Diseases, 72(1), 9-14.

Braun, V., \& Clarke, V. (2006). Using thematic analysis in psychology. Qualitative Research in Psychology, 3(2), 77-101.
Centers for disease control and prevention. (2017a). HIV surveillance report, 2016, vol. 28. https://www.cdc.gov/hiv/pdf/library/repor ts/surveillance/cdc-hiv-surveillance-report-2016-vol-28.pdf

Centers for Disease Control and Prevention. (2017b). Recommendations for HIV screening of gay, bisexual, and other men who have sex with men: United States, 2017, vol. 66. https://www.cdc.gov/ mmwr/volumes/66/wr/mm6631a3.htm.

Centers for Disease Control and Prevention. (2017c). Pre-exposure prophylaxis for the prevention of HIV infection in the United States, 2017, vol. 66. https://www.cdc.gov/hiv/pdf/risk/prep/ cdc-hiv-prep-guidelines-2017.pdf.

Centers for Disease Control and Prevention. (2018). Monitoring selected national HIV prevention and care objectives by using HIV surveillance data: United States and 6 dependent areas, 2016. https://www.cdc.gov/hiv/library/reports/hiv-surveillan ce.html.

Centers for Disease Control and Prevention. (2020). PrEP During COVID-19. https://www.cdc.gov/nchhstp/dear_colleague/2020/ dcl-051520-PrEP-during-COVID-19.html.

Doll, M., Fortenberry, J. D., Roseland, D., McAuliff, K., Wilson, C. M., \& Boyer, C. B. (2018). Linking HIV-negative youth to prevention services in 12 US cities: Barriers and facilitators to implementing the HIV prevention continuum. Journal of Adolescent Health, 62(4), 424-433.

Hill, B. J., Anderson, B., \& Lock, L. (2021). COVID-19 pandemic, PreExposure Prophylaxis (PrEP) Care, and HIV/STI testing among patients receiving care in three HIV epidemic priority states. AIDS and Behavior, 25(5), 1361-1365.

Holloway, I. W., Dougherty, R., Gildner, J., Beougher, S. C., Pulsipher, C., Montoya, J. A., Plant, A., \& Leibowitz, A. (2017). Brief report: PrEP uptake, adherence, and discontinuation among california YMSM using geosocial networking applications. Journal of Acquired Immune Deficiency Syndromes, 74(1), 15-20.

Hong, C., Horvath, K. J., Stephenson, R., Nelson, K. M., Petroll, A. E., Walsh, J. L., \& John, S. A. (2021). PrEP use and persistence among young sexual minority men 17-24 years old during the COVID-19 pandemic. AIDS and Behavior, 1-8.

Jenness, S. M., Le Guillou, A., Chandra, C., Mann, L. M., Sanchez, T., Westreich, D., \& Marcus, J. L. (2020). Projected HIV and bacterial STI incidence following COVID-related sexual distancing and clinical service interruption. medRxiv.

John, S. A., Rendina, H. J., Grov, C., \& Parsons, J. T. (2017). Homebased pre-exposure prophylaxis (PrEP) services for gay and bisexual men: an opportunity to address barriers to PrEP uptake and persistence. PLoS One, 12(12), e0189794.

John, S. A., Robles, G., Starks, T. J., \& Rendina, H. J. (2019). Differences between groups of pre-exposure prophylaxis (PrEP) using couples in HIV-negative/unknown relationships. Journal of Acquired Immune Deficiency Syndromes, 81(4), 419.

John, S. A., Sizemore, K. M., Jimenez, R. H., Jones, S. S., Petroll, A. E., \& Rendina, H. J. (2021). PEP as a potential antecedent to PrEP among an online sample of HIV-negative and unknown status cis- and transgender sexual minority men: A cross-sectional study. JMIR Preprints., 15(06/2021), 31237.

John Hopkins Coronavirus Resource Center. (n.d.). United States cases by county. Johns Hopkins University \& Medicine. Retrieved February 24, 2021, from https://coronavirus.jhu.edu/us-map.

Junejo, M., Girometti, N., McOwan, A., Whitlock, G., Gedela, K., McCormack, S., Nugent, D., Patel, S., Suchak, T., \& Tittle, V. (2020). HIV postexposure prophylaxis during COVID-19. The Lancet HIV, 7(7), e460.

Kneale, D., \& Becares, L. (2020). The mental health and experiences of discrimination of LGBTQ + people during the COVID-19 pandemic: Initial findings from the Queerantine Study. MedRxiv.

Koechlin, F. M., Fonner, V. A., Dalglish, S. L., O'Reilly, K. R., Baggaley, R., Grant, R. M., Rodolph, M., Hodges-Mameletzis, L., \& 
Kennedy, C. E. (2017). Values and preferences on the use of oral pre-exposure prophylaxis (PrEP) for HIV prevention among multiple populations: A systematic review of the literature. AIDS and Behavior, 21(5), 1325-1335.

Mann, D. M., Chen, J., Chunara, R., Testa, P. A., \& Nov, O. (2020). COVID-19 transforms health care through telemedicine: Evidence from the field. Journal of the American Medical Informatics Association, 27(7), 1132-1135.

Mayer, K. H., Jones, D., Oldenburg, C., Jain, S., Gelman, M., Zaslow, S., \& Mimiaga, M. J. (2017). Excellent HIV post-exposure prophylaxis regimen completion with single tablet daily elvitegravir/ cobicistat/tenofovir disoproxil fumarate/emtricitabine compared to more frequent dosing regimens. Journal of Acquired Immune Deficiency Syndromes, 75(5), 535.

Nelson, K. M., Gordon, A. R., John, S. A., Stout, C. D., \& Macapagal, K. (2020). Physical sex is over for now: Impact of COVID-19 on the well-being and sexual health of adolescent sexual minority males in the US. Journal of Adolescent Health, 67(6), 756-762.

Pampati, S., Emrick, K., Siegler, A. J., \& Jones, J. (2021). Changes in sexual behavior, PrEP adherence, and access to sexual health services because of the COVID-19 pandemic among a cohort of PrEP-using MSM in the south. Journal of Acquired Immune Deficiency Syndromes, 87(1), 639-643.

Quinn, K., Dickson-Gomez, J., Zarwell, M., Pearson, B., \& Lewis, M. (2019). "A gay man and a doctor are just like, a recipe for destruction": How racism and homonegativity in healthcare settings influence PrEP uptake among young Black MSM. AIDS and Behavior, 23(7), 1951-1963.

Reisner, S. L., Randazzo, R. K., White Hughto, J. M., Peitzmeier, S., DuBois, L. Z., Pardee, D. J., Marrow, E., McLean, S., \& Potter, J. (2018). Sensitive health topics with underserved patient populations: Methodological considerations for online focus group discussions. Qualitative Health Research, 28(10), 1658-1673.

Richards, M., Anderson, M., Carter, P., Ebert, B. L., \& Mossialos, E. (2020). The impact of the COVID-19 pandemic on cancer care. Nature Cancer, 1(6), 565-567.

Ridgway, J. P., Schmitt, J., Friedman, E., Taylor, M., Devlin, S., McNulty, M., \& Pitrak, D. (2020). HIV care continuum and COVID-19 outcomes among people living with HIV during the COVID-19 pandemic, Chicago, IL. AIDS and Behavior, 24, $2770-2772$.

Rogers, B. G., Tao, J., Maynard, M., Chu, C., Silva, E., Toma, E., Nagel, K.,, Napoleon S., \& Chan, P. A. (2021). Characterizing the impact of COVID-19 on pre-exposure prophylaxis (PrEP) care. AIDS and Behavior, 1-4.

Saldaña, J. (2014). Thinking qualitatively: Methods of mind. Sage Publications.

Sanchez, T. H., Zlotorzynska, M., Rai, M., \& Baral, S. D. (2020). Characterizing the Impact of COVID-19 on Men Who Have Sex with Men Across the United States in April 2020. AIDS and Behavior, 24(7), 2024-2032.

Shoptaw, S., Goodman-Meza, D., \& Landovitz, R. J. (2020). Collective call to action for HIV/AIDS community-based collaborative science in the era of COVID-19. AIDS and Behavior, 24(7), 2013-2016.

Siegler, A. J., Bratcher, A., Weiss, K. M., Mouhanna, F., Ahlschlager, L., \& Sullivan, P. S. (2018). Location location location: An exploration of disparities in access to publicly listed pre-exposure prophylaxis clinics in the United States. Annals of Epidemiology, 28(12), 858-864.

Skarbinski, J., Rosenberg, E., Paz-Bailey, G., Hall, H. I., Rose, C. E., Viall, A. H., Fagan, J. L., Lansky, A., \& Mermin, J. H. (2015). Human immunodeficiency virus transmission at each step of the care continuum in the United States. JAMA Internal Medicine, 175(4), 588-596.

Smith, D. K., Grohskopf, L. A., Black, R. J., Auerbach, J. D., Veronese, F., Struble, K. A., Cheever, L., Johnson, M., Paxton, L. A., Onorato, I. M., \& Greenberg, A. E. (2005). Antiretroviral postexposure prophylaxis after sexual, injection drug use, or other nonoccupational exposure to HIV in the United States: Recommendations from the US Department of Health and Human Services. Morbidity and Mortality Weekly Report: Recommendations and Reports, $54(2), 1-20$.

Stephenson, R., Chavanduka, T. M., Rosso, M. T., Sullivan, S. P., Pitter, R. A., Hunter, A. S., \& Rogers, E. (2021). Sex in the time of COVID-19: Results of an online survey of gay, bisexual and other men who have sex with men's experience of sex and HIV prevention during the US COVID-19 epidemic. AIDS and Behavior, 25(1), 40-48.

Strauss, A., \& Corbin, J. M. (1997). Grounded theory in practice. Sage. Teitcher, J. E., Bockting, W. O., Bauermeister, J. A., Hoefer, C. J., Miner, M. H., \& Klitzman, R. L. (2015). Detecting, preventing, and responding to "fraudsters" in internet research: Ethics and tradeoffs. The Journal of Law, Medicine \& Ethics, 43(1), 116-133.

Touger, R., \& Wood, B. R. (2019). A review of telehealth innovations for HIV pre-exposure prophylaxis (PrEP). Current HIV/AIDS Reports, 16(1), 113-119.

U.S. Food \& Drug Administration. (2019). FDA approves second drug to prevent HIV infection as part of ongoing efforts to end the HIV epidemic. Retrieved February 24, 2021, from https://www.fda.gov/ news-events/press-announcements/fda-approves-second-drugp revent-hiv-infection-part-ongoing-efforts-end-hiv-epidemic.

U.S. Food and Drug Administration. (2012). FDA approves first medication to reduce HIV risk. Retrieved February 24, 2021, from: https://wayback.archiveit.org/7993/20170406045106/https://www. fda.gov/ForConsumer/ConsumerUpdates/ucm311821.htm.

Woodyatt, C. R., Finneran, C. A., \& Stephenson, R. (2016). In-person versus online focus group discussions: A comparative analysis of data quality. Qualitative Health Research, 26(6), 741-749.

Ya-lin, A. H., Zhu, W., Smith, D. K., Harris, N., \& Hoover, K. W. (2018). HIV preexposure prophylaxis, by race and ethnicity: United States, 2014-2016. Morbidity and Mortality Weekly Report, 67(41), 1147.

Zapata, J. P., Petroll, A., de St-Aubin, E., \& Quinn, K. (2020). Perspectives on social support and stigma in PrEP-related care among gay and bisexual men: A qualitative investigation. Journal of Homosexuality. https://doi.org/10.1080/00918369.2020.1819709

Zarwell, M., John, S. A., Westmoreland, D., Mirzayi, C., Pantalone, D. W., Golub, S., Nash, D., \& Grov, C. (2020). PrEP uptake and discontinuation among a US national sample of transgender men and women. AIDS and Behavior, 1-9.

Publisher's Note Springer Nature remains neutral with regard to jurisdictional claims in published maps and institutional affiliations. 